\title{
Expression of hepatocyte growth factor activator inhibitors (HAI-1 and HAI-2) in ovarian cancer
}

\author{
KEIICHIRO NAKAMURA ${ }^{1}$, FERNANDO ABARZUA ${ }^{2}$, JUNICHI KODAMA ${ }^{1}$, ATSUSHI HONGO ${ }^{1}$, \\ YASUTOMO NASU $^{2}$, HIROMI KUMON ${ }^{2}$ and YUJI HIRAMATSU ${ }^{1}$ \\ Departments of ${ }^{1}$ Obstetrics and Gynecology, ${ }^{2}$ Urology, Okayama University Graduate School of Medicine, \\ Dentistry and Pharmaceutical Sciences, Okayama, Japan
}

Received August 25, 2008; Accepted October 24, 2008

DOI: 10.3892/ijo_00000157

\begin{abstract}
In light of the poor prognosis for ovarian cancer, research continues for innovative and efficacious treatment modalities. It is now widely accepted that new approaches for the treatment of ovarian cancers are pivotal to further improve the prognosis of this disease. We investigated the role of HAI-1 and HAI-2, and evaluated their clinical importance in ovarian cancer. The distribution of cases that scored positive for each of the biological parameters examined was correlated with HAI-1 and HAI-2 expression status obtained by immunohistochemistry. There was a significant correlation between HAI-2 expression and stage ( $\mathrm{p}=0.031)$, amount of ascites $(\mathrm{p}=0.002)$ and diameter of residual tumor $(\mathrm{p}=0.034)$. HAI-1 expression was a significant associated with stage $(\mathrm{p}=0.040)$. Furthermore, the low HAI-1 and HAI-2 expression was a significant predictor for poor prognosis when compared with high HAI-1 and HAI-2 expression (disease-free survival rate; $\mathrm{p}=0.031$ and $\mathrm{p}=0.003$, overall survival rate; $\mathrm{p}=0.048$ and $\mathrm{p}=0.001)$. Therefore, we investigated biological functions and effects of HAI- 1 and HAI-2 using OVCAR-3 ovarian cancer cell lines. HAI-1 and HAI-2 show potential inhibitory effects mediated by reduction of matriptase and hepsin expression which leads to apoptosis through increasing the
\end{abstract}

Correspondence to: Dr Keiichiro Nakamura, Department of Obstetrics and Gynecology, Okayama University Graduate School of Medical, Dentistry and Pharmaceutical Sciences, 2-5-1 Shikatacho, Okayama 700-8558, Japan

E-mail: k-nakamu@cc.okayama-u.ac.jp

Abbreviations: ECM, extracellular matrix; HGF, hepatocyte growth factor; HGFA, HGF activator; HAI-1, HGFA inhibitor type I; HAI-2, HGFA inhibitor type II; PB, placental bikunin; PI, propidium iodide; DFS, disease-free survival; OS, overall survival; uPA, urokinase plasminogen activator; DMEM, Dulbecco's modified Eagle's medium; FIGO, international federation of gynecology and obstetrics; FBS, fetal bovine serum

Key words: HAI-1, HAI-2, ovarian cancer, favorable prognosis marker, apoptotic pathway level of Bak and reducing the level of Bcl-2 on ovarian cancer. These findings indicate that low HAI-1 and HAI-2 expression in ovarian cancer may be associated with poor prognosis. HAI-1 and HAI-2 could be considered a therapeutic target for treatment approaches in ovarian cancer.

\section{Introduction}

Despite its pervasive impact, the etiology of ovarian cancer and the factors that promote its progression are not yet well understood. It is now widely accepted that new approaches for the treatment of ovarian cancers are pivotal in further improving prognosis of this disease.

Hepatocyte growth factor (HGF) is secreted from mesenchymal cells of the liver as an inactive singlechain form and normally remains in this form associated with the extracellular matrix (ECM) (1). It has mitogentic, motogenic, morphogenic functions in various types of cells through its high-affinity receptor tyrosine kinase that is encoded by the c-Met proto-oncogene. The c-Met have important roles in migration, invasion of tumor cells, differentiation, and tumor angiogenesis $(2,3)$. The c-Met overexpression in ovarian cancer is associated with ovarian cancer progression, and high c-Met expression correlates with poor survival $(4,5)$.

$\mathrm{HGF}$ is secreted as an inactive pro-peptide which is cleaved by HGF activator (HGFA) into its active form. HGFA is regulated by two inhibitors, HGFA inhibitor type I (HAI-1) and type II (HAI-2) they are type I transmembrane proteins with two Kunitz-type serine protease domains in the extraxellular portion, and a cell surface expression on epithelial cells (6-9). Among HAI-1 and HAI-2, membraneform HAI-1 can bind to HGFA on the cellular surface, but membrane-form HAI-2 cannot (10). It is thus very likely that HAI-1 and HAI-2 have distinct biological roles in vivo. The binding of HGFA to HAI- 1 is reversible and may reserve available HGFA on the cell surface to ensure concentrated pericellular HGFA activity (11). HAI-1 appears to be the cognate inhibitor of HGFA and matriptase, and both HGFA and hepsin $(11,12)$. HAI-2 is a more efficient inhibitor of hepsin and displays a broader inhibitory spectrum than HAI-1 (13). To date, several studies on HAI-1 and HAI-2 expression in tumor tissues have been published. The loss of 
HAI-1 expression has been implicated in the progression of breast, prostate, renal and colorectal carcinoma in humans (14-17). The matriptase to HAI-1 ratio could be important in the development of advance disease on ovarian cancer (18). One study concluded that HAI-2 expression was up-regulated in pancreatic cancers (9), whereas another demonstrated down-regulation in glioblastoma, hepatocellular carcinomas and renal carcinoma $(10,19,20)$.

To our knowledge, HAI-1 and HAI-2 have not been reported in the same patient with ovarian cancer. In the current study, we examined whether HAI-1 and HAI-2 protein expression correlate with clinicopathological characteristics in patients suffering from ovarian cancer. The main aim of the study was to determine whether HAI- 1 and HAI-2 protein could be used as an early invasive marker for patients and considered a therapeutic target for treatment approaches in ovarian cancer.

\section{Materials and methods}

Patients and tissues. Patients with malignant epithelial ovarian tumor $(n=54)$ were treated at Okayama University Hospital. All specimens were obtained at the time of surgery from the primary lesion. Informed consent was obtained from each patient before sample collection. The histological malignant cell types of tumors were assigned according to the WHO classification: 32 were classified as serous cell type, 10 as endometrioid cell type, 2 as mucinous cell type and 10 as clear cell type. Surgical staging was reviewed based on the International Federation of Obstetrics and Gynecology (FIGO) staging system: 17 were stage I, 5 were stage II, 28 were stage III and 4 were stage IV. Overall survival was defined as the interval from the initial surgery to death or to March 30, 2006. The median duration of follow-up was 48 months (range 6-146). Tumor specimens were fixed in $10 \%$ neural-buffered formalin immediately after excision, dehydrated through graded series of ethanol and xylene, embedded in paraffin.

Immunohistochemistry and staining evaluation. Formalinfixed, paraffin-embedded sections, $4 \mu \mathrm{m}$ thick, were deparaffinized with xylene and rehydrated in ethanol. Endogenous peroxidase activity was quenched by methanol containing $0.3 \%$ hydrogen peroxidase for $15 \mathrm{~min}$. Then, sections were incubated at room temperature with a HAI-1 and HAI-2 (Santa Cruz Biotechnology, Santa Cruz, CA) followed by staining using a streptavidin-biotin-peroxidase kit (Nichirei, Tokyo, Japan). The sections were counterstained with hematoxylin. The level of HAI-1 or HAI-2 staining in epithelial cells was classified into three groups by scoring the percentage of positive cells: strong (2); $>50 \%$ of cells stained, moderate (1); $10-50 \%$ of cells stained, and weak (0); $<10 \%$ of cells stained. Microscopic analyses were independently conducted by two examiners with no prior knowledge of the clinical data. Final decisions in controversial cases were made using a conference microscope.

Cell culture, media and generation of transfectants. OVCAR-3 (ATCC Number: HTB-161) cancer cell lines were derived from human ovarian carcinomas. OVCAR-3 ovarian cancer cells were maintained in Dulbecco's modified Eagle's medium (DMEM) with supplemented with $10 \%$ charcoal-striped fetal bovine serum (FBS). Cells were maintained at $37^{\circ} \mathrm{C}$ in an atmosphere with $5 \% \mathrm{CO}_{2}$ in air. A cDNA encoding the whole coding region of HAI-1 or HAI2/PB was constructed by polymerase chain reaction using full-length HAI-1 cDNA or HAI-2 cDNA as a template. Primers used were: 5'-TTGGAATTCGCGATGGCCCCTG CGAGGAC-3' and 5'-TTAGTCGACTCAGAGGGGCCGG GTG GTGT-3' for HAI-1, and 5'-AGCTCTAGAGCCA TGGCGCAGCTGTGCGG-3' and 5'-TTAGTCGACTCA CAGGACATATGTGTTCTTC-3' for HAI-2/PB. The polymerase chain reaction products were subcloned into the EcoRI/SalI site (HAI-1) or XbaI/SalI site (HAI-2/PB) of an expression plasmid pCIneo (Promega, Madison, WI). HAI-1 and HAI-2 cDNA expression vector was transient transfected into OVCAR-3 ovarian cancer cells using TransFast transfection reagent (Promega). Mock transfected cells served as a control.

Western blot analysis. Cell lysates were collected and estimated using a Protein Assay system (Bio-Rad, Hercules, CA) according to the manufacturer's protocols. Proteins from each cell line were subjected to SDS-PAGE, and were transferred onto a nitrocellulose membrane. Polyclonal and monoclonal antibodies used for immunoblotting were as follows: HAI-1, HAI-2, Matriptase, Bcl-2, Bak (Santa Cruz Biotechnology), Hepsin (Cayman Chemical, Ann Arbor, MI), and B-actin (Sigma Chemical, St. Louis, MO). Working dilution for the primary antibobies was 1:1000. Membranes were then incubated with appropriate secondary antibodies. Expressions of antigen-antibody complexes were detected with an enhanced chemiluminesence kit (Amershan Biosciences, Piscataway, NJ).

Cell viability assay. To examine the cell viability by transient transfection with HAI-1 or HAI-2 in OVCAR-3 cells SYTO 10 green fluorescent nucleic acid stain and Dead red (ethidium homodimer-2) nucleic acid stain (Live/Dead ${ }^{\circledR}$ reduced biohazard viability/cytotoxicity kit; Invitrogen, Eugene, OR) were added to each well and incubated for 15 min. Cell fluorescence was observed by a fluorescent microscope (Olympus, Tokyo, Japan).

Matrigel invasion assay. To investigate differences in invasive ability between cells expressing HAI-1 or HAI-2, we used BD BioCoat Matrigel Invasion Chamber (BD Bioscience, Bedford, MA). Either with or without transient transfection with HAI-1 or HAI-2 in OVCAR-3 ovarian cancer cells were added in situ with $10 \mu \mathrm{g} / \mathrm{ml}$ of DiI (Invitrogen, Carlsbad, CA) in DMEM with 10\% FBS. Containing 10\% FBS for $1 \mathrm{~h}$. Cells $\left(5 \times 10^{4}\right)$ of each genotype were added to inserts, and $0.75 \mathrm{ml}$ of medium was added to the bottom of each well. After 12, 24, 36 and $48 \mathrm{~h}$ of incubation, membranes were removed from the insert and mounted on slides, and then invading cells were counted under the microscope. Matrigel assays were performed in triplicate.

MTS assay and FITC-conjugated Annexin V. In order to evaluate the cell proliferation effects of HAI-1 and HAI-2, 
A

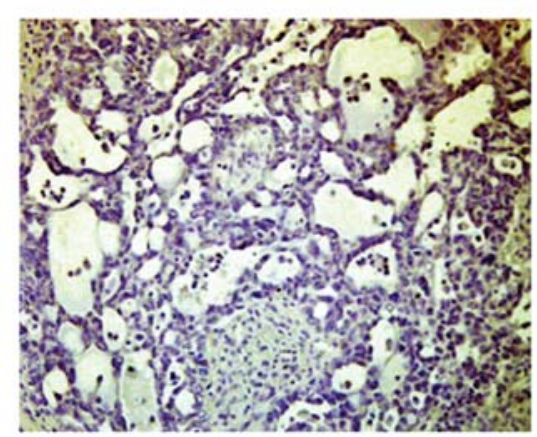

D

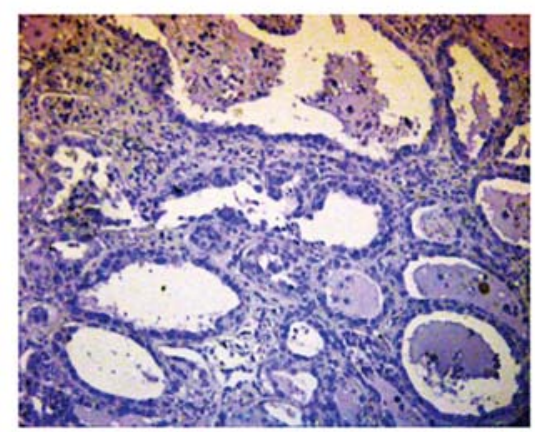

B

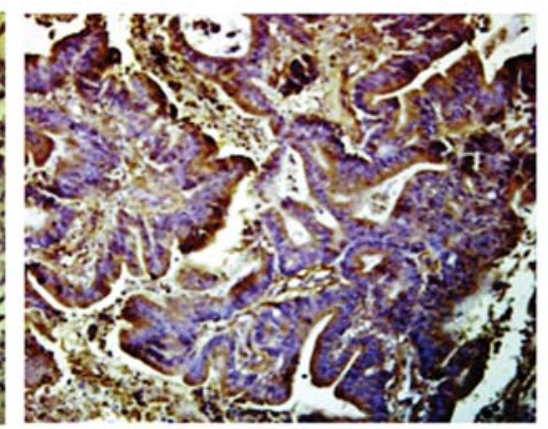

E

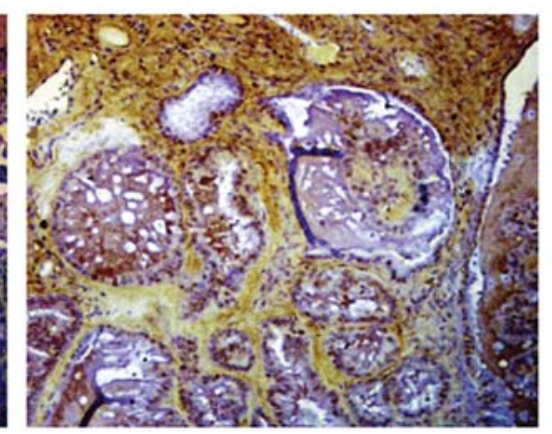

C

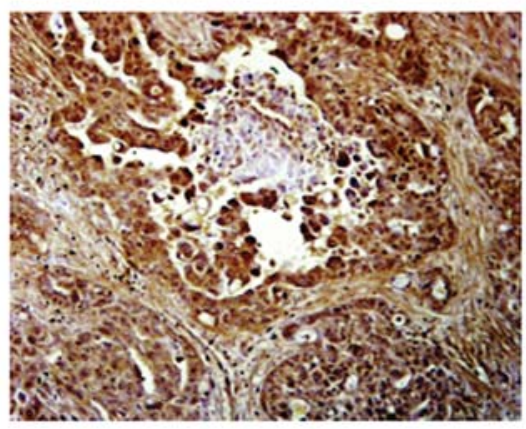

F

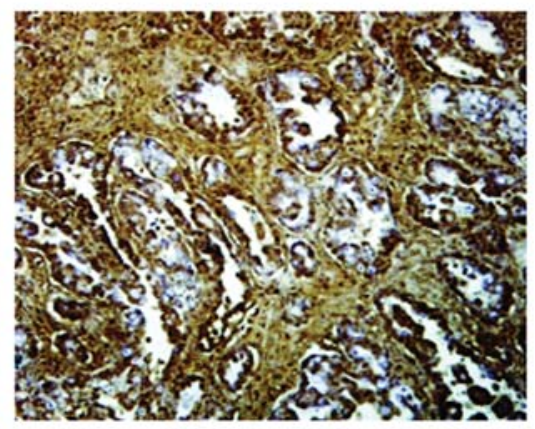

Figure 1. Representative immunostaining patterns of HAI-1 (A) weak epithelial cell staining. (B) Moderate epithelial cell staining. (C) Strong epithelial cell staining. Representative immunostaining patterns of HAI-2. (D) Weak epithelial cell staining. (E) Moderate epithelial cell staining. (F) Strong epithelial cell staining (original magnification x100). The relative strength of HAI-1 and HAI-2 immunohistochemical staining was assessed qualitatively.

MTS assay (Promega) was performed. Cells were seeded into 96-well plates to obtain a density of $5 \times 10^{4}$ cells/well and transfected. Cells were treated either with or without transient transfection with HAI-1 or HAI-2 treatment for 12, 24, 36 and $48 \mathrm{~h}$ and then MTS was added for $2 \mathrm{~h}$. The absorbance was read at a wavelength of $490 \mathrm{~nm}$ using an ELISA platereader (Bio-Rad systems). Moreover, apoptosis was measured by staining with FITC-conjugated Annexin V using a MEBCyto Apoptosis Kit (MBL, Nagoya, Japan) according to the manufacturer's recommendations with flow cytometric analysis.

Cell growth in monolayers and in soft agar. In monolayers of cell growth, cells were plated at a density of $3 \times 10^{4}$ cells/ well in 6-well plates containing DMEM with $10 \%$ FBS. Cell proliferations were assessed by counting the cell numbers in triplicate after 2, 4, 6, 8 and 10 days using a hemocytometer. In soft agar, a cell suspension ( $1 \times 10^{4}$ cells/well) in $1 \mathrm{ml} 0.2 \%$ Noble agar DMEM with $10 \%$ FBS was overlaid onto a 35 mm dish containing a $0.5 \%$-agar base. Colonies $>0.2 \mathrm{~mm}$ in diameter were counted on the 21 st day after culture. Soft agar assays were performed in triplicate.

Statistical analysis. Statistical analyses were performed using the Mann-Whitney U test for comparison with the control and one-factor ANOVA followed by Fisher's protected least significance difference test for all pairwise comparisons. The analyses were performed with the software package StatView version 5.0 (Abacus Concepts, Berkeley, CA). Differences were considered significant at $\mathrm{p}<0.05$.

\section{Results}

Expression of HAI-1 and HAI-2 were examined in human ovarian tissues by immunostaining. Expression of HAI-1 and HAI-2 were examined in human epithelial ovarian cancer tissues by immunostaining; Fig. 1 illustrates representative immunostaining patterns of HAI-1 and HAI-2. Weak epithelial staining was observed respectively in 9 cases $(16.6 \%)$ and 15 cases $(27.8 \%)$, moderate staining in 30 cases $(55.6 \%)$ and 20 cases $(37.0 \%)$, and strong staining in 15 cases $(27.8 \%)$ and 19 cases $(35.2 \%)$ of HAI- 1 and HAI- 2 .

The biological parameters examined by immunostaining patterns of HAI-1 and HAI-2. Table I shows the distribution of cases scored as positive for each of the biological parameters examined, according to clinicopathological characteristics in the overall population. As expected, the expression of HAI-2 has a statistically significant association with clinicopathological parameters such as stage $(\mathrm{p}=0.031)$, amount of ascites $(\mathrm{p}=0.002)$, diameter of residual of tumor size $(\mathrm{p}=0.034)$. The expression of HAI- 1 shows a statistically significant association only with stage $(\mathrm{p}=0.040)$ (MannWhitney U test, $\mathrm{p}<0.05$ ) (Table I).

The disease-free and overall survival analysis. HAI-1 and HAI-2 were significant in the disease-free survival (DFS) and overall survival (OS) analysis of prognostic factor using the log-rank test in ovarian cancer. Fig. 2 shows DFS and OS curves of 54 patients with ovarian cancer, according to HAI-1 and HAI-2 expression status. The DFS and OS rates 
Table I. Association between HAI-1 or HAI-2 and clinicopathological factors in ovarian cancer.

\begin{tabular}{|c|c|c|c|c|c|}
\hline Variable & $\begin{array}{l}\text { No. of cases } \\
\qquad(n=54)\end{array}$ & $\begin{array}{c}\text { HAI-1 } \\
(\text { mean } \pm \mathrm{SE})\end{array}$ & P-value ${ }^{a}$ & $\begin{array}{c}\text { HAI-2 } \\
(\text { mean } \pm \mathrm{SE})\end{array}$ & P-value ${ }^{a}$ \\
\hline Age (years) & & & 0.186 & & 0.836 \\
\hline$\leq 60$ & 39 & $1.05 \pm 0.69$ & & $1.08 \pm 0.81$ & \\
\hline$>60$ & 15 & $1.27 \pm 0.59$ & & $1.13 \pm 0.74$ & \\
\hline Stage & & & $0.040^{\mathrm{a}}$ & & $0.031^{\mathrm{a}}$ \\
\hline I + II & 21 & $1.35 \pm 0.67$ & & $1.38 \pm 0.74$ & \\
\hline III + IV & 33 & $0.97 \pm 0.63$ & & $0.91 \pm 0.77$ & \\
\hline Grade & & & 0.262 & & 0.276 \\
\hline $1+2$ & 21 & $1.24 \pm 0.54$ & & $1.24 \pm 0.83$ & \\
\hline $3 / \mathrm{NE}$ & 33 & $1.03 \pm 0.73$ & & $1.00 \pm 0.75$ & \\
\hline Histological type & & & 0.139 & & 0.495 \\
\hline Non-serous & 22 & $1.27 \pm 0.63$ & & $1.18 \pm 0.80$ & \\
\hline Serous & 32 & $1.00 \pm 0.66$ & & $1.03 \pm 0.78$ & \\
\hline Amount of ascites (ml) & & & 0.672 & & $0.002^{\mathrm{a}}$ \\
\hline$<1000$ & 34 & $1.14 \pm 0.67$ & & $1.30 \pm 0.74$ & \\
\hline$\geq 1000$ & 20 & $1.06 \pm 0.66$ & & $0.65 \pm 0.70$ & \\
\hline Diameter of residual tumor size & & & 0.639 & & $0.034^{\mathrm{a}}$ \\
\hline$\leq 2 \mathrm{~cm}$ & 35 & $1.14 \pm 0.65$ & & $1.26 \pm 0.74$ & \\
\hline$>2 \mathrm{~cm}$ & 19 & $1.05 \pm 0.71$ & & $0.79 \pm 0.79$ & \\
\hline Lymph node metastasis ${ }^{b}$ & & & 0.164 & & 0.499 \\
\hline Negative & 33 & $1.21 \pm 0.70$ & & $1.15 \pm 0.80$ & \\
\hline Positive & 21 & $0.95 \pm 0.59$ & & $1.00 \pm 0.77$ & \\
\hline
\end{tabular}

${ }^{a}$ Mann-Whitney U test. ${ }^{b}$ Lymph node status was assessed by CT imaging in 23 patients.

of patients exhibiting high HAI-1 and HAI-2 expression (score 2) were significantly higher than those of patients exhibiting low HAI-1 and HAI-2 expression (score 0-1). ( $\mathrm{p}=0.031$ and $\mathrm{p}=0.003, \mathrm{p}=0.048$ and $\mathrm{p}=0.001$, respectively) (Mann-Whitney U test, $\mathrm{p}<0.05$ ).

The protein expression of HAI-1, HAI-2, hepsin and matriptase by transient transfection with HAI-1 or HAI-2 into OVCAR-3 ovarian cell lines. We examined the functional role of HAI-1 and HAI-2 which were generated as described in Materials and methods. As shown in Fig. 3A, HAI-1 and HAI-2 levels were almost absent in the OVCAR-3 cancer cell lines. HAI-1 and HAI-2 protein levels were highly expressed in the transient transfection with HAI-1 and HAI-2. The expression of matriptase and hepsin was decreased by transient transfection with both HAI-1 and HAI-2. Furthermore, matriptase was inhibited more than hepsin by HAI-1 and HAI-2.

Cell viability by transient transfection with HAI-1 or HAI-2 into OVCAR-3 ovarian cancer cell lines. We investigated the efficacy of cell viability of OVCAR-3 ovarian cancer cells by transient transfection with HAI-1 or HAI-2. The percentage of cell viability was decreased to $35.6 \%$ and
$48.8 \%$ of control at $48 \mathrm{~h}$ after transient transfection with HAI-1 or HAI-2 into OVCAR-3 cell lines, respectively. Therefore, the dead cells were increased by transient transfection with HAI-1 and HAI-2 into OVCAR-3 cells (Fig. 3B).

Matrigel invasion of transient transfection with HAI-1 or HAI-2 into OVCAR-3 cells. We assessed motility and invasiveness of cells overexpressing HAI-1 and HAI-2 using a BioCoat Matrigel Invasion Chamber. Cells were plated on the upper surface of the membrane, cells on the bottom of the membrane were stained and counted. The percentage of cells reaching the bottom of the filter was decreased to $90.6 \%$, $86.6 \%$ of control at $12 \mathrm{~h}, 64.2 \%, 58.4 \%$ at $24 \mathrm{~h}, 37.6 \%, 32.2 \%$ at $36 \mathrm{~h}$, and $29.8 \%, 25.5 \%$ at $48 \mathrm{~h}$ after transient transfection of HAI-1 or HAI-2 into OVCAR-3 cells, respecting (Fig. 4A).

Apoptosis of the transient transfection with HAI-1 or HAI-2 into OVCAR-3 cell lines. We used MTS and FITC-conjugated Annexin $\mathrm{V}$ assays that examined apoptosis expression by transient transfection of HAI-1 or HAI-2 into OVCAR-3 ovarian cell lines. The transient transfection of HAI-1 and HAI-2 cased reduced cell growth in MTS assay (Fig. 4B). Representative flow cytometric data revealed that transient 
A

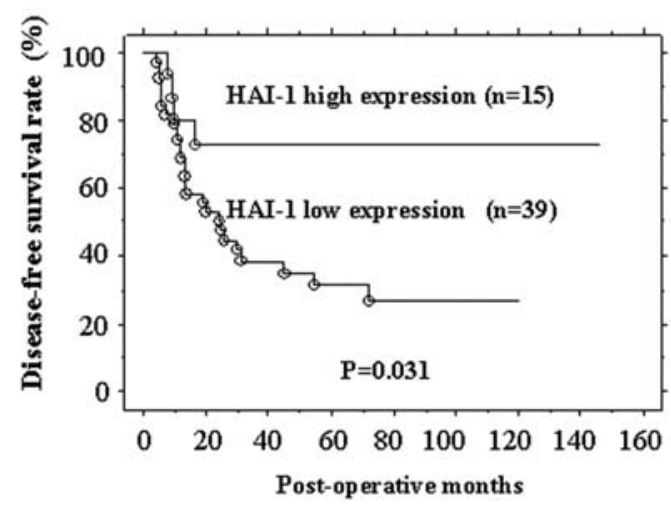

C

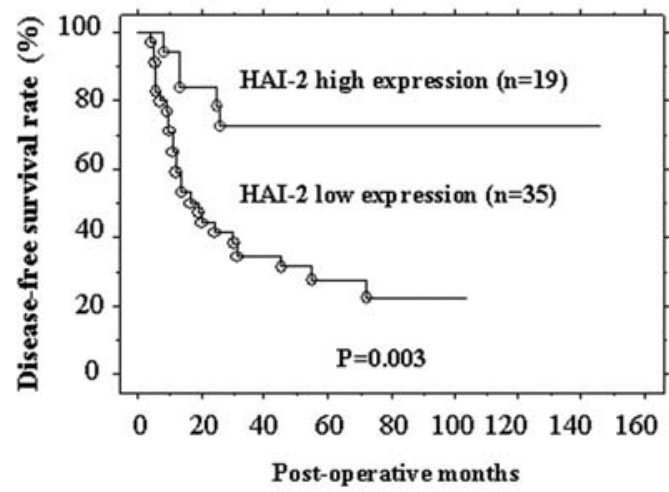

B

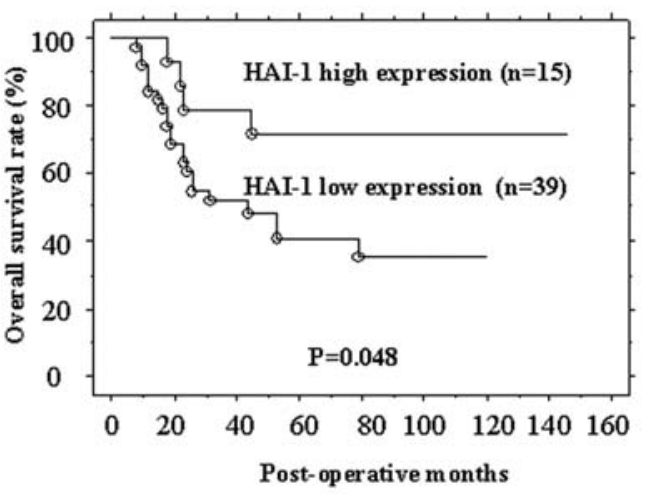

D

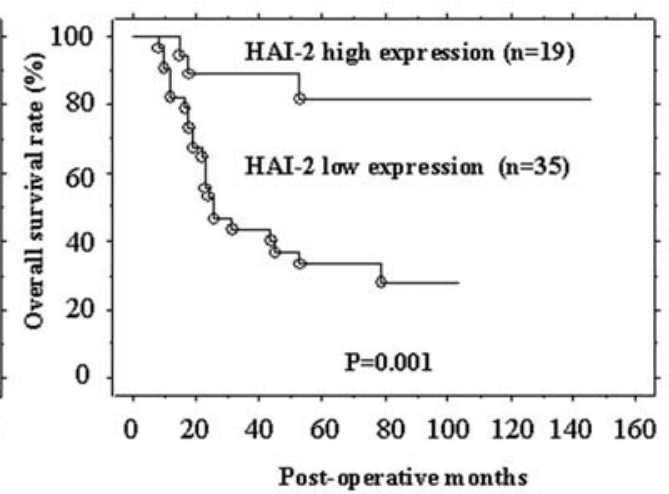

Figure 2. Kaplan-Meier plots for (A) disease-free and (B) overall survival of the 54 patients with ovarian cancer according to their epithelial HAI-1 expression status. Kaplan-Meier plots for (C) disease-free and (D) overall survival of the 54 patients with ovarian cancer according to their epithelial HAI-2 expression status. Low epithelial expression, score 0-1; high epithelial expression, score 2.

A 1. $2 . \quad 3$.

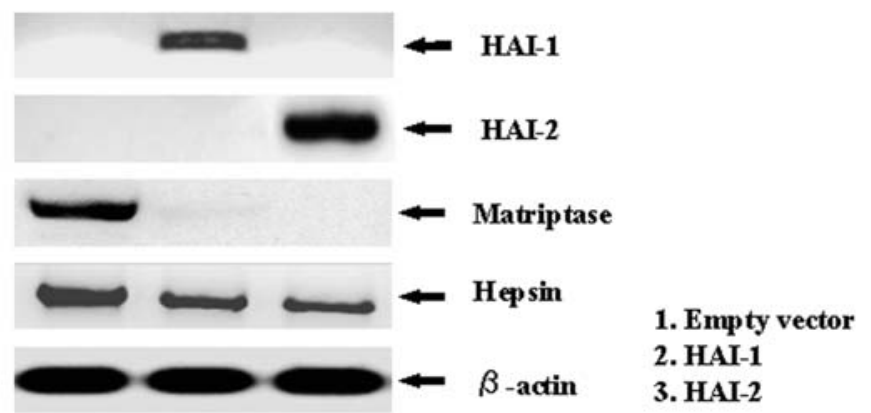

B

Empty vector

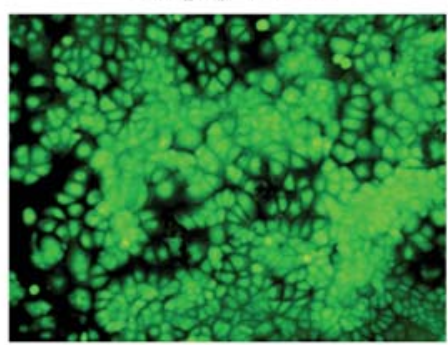

HAI-1

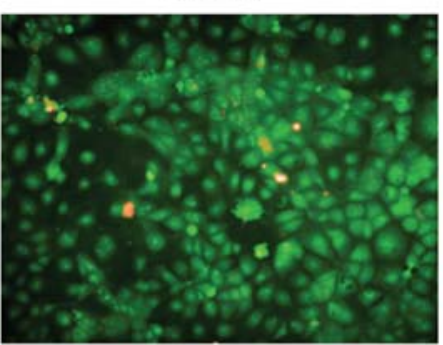

HAI-2

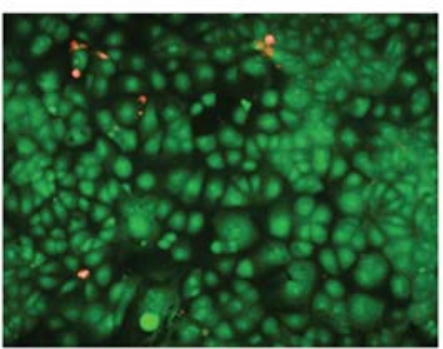

Figure 3. Evaluation of transient transfection of HAI-1 and HAI-2 into OVCAR-3 ovarian cancer cells. (A) The transient transfection of HAI-1 or HAI-2 into OVCAR-3 ovarian cancer cells were analyzed for HAI-1, HAI-2, matriptase, and hepsin expression by Western blotting. B-actin antibody was used as loading control in the same blot. (B) The cell viability was examined by transient transfection with HAI-1 or HAI-2 into OVCAR-3 ovarian cancer cell lines. Cell viability was analyzed by a fluorescent microscope. 
A

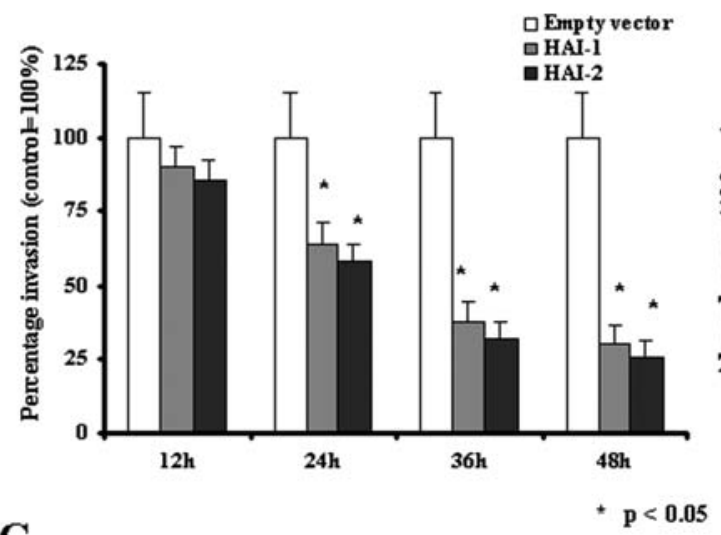

C

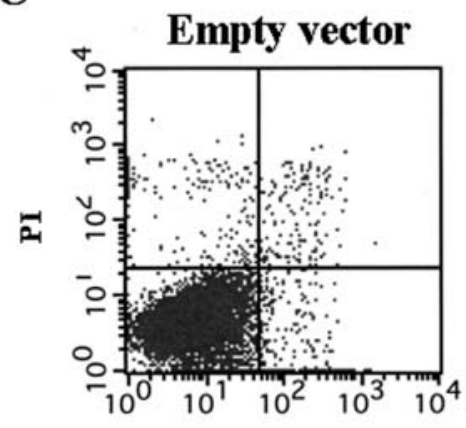

Annexin-V

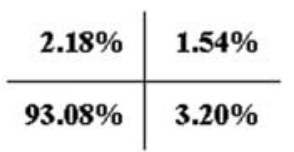

B

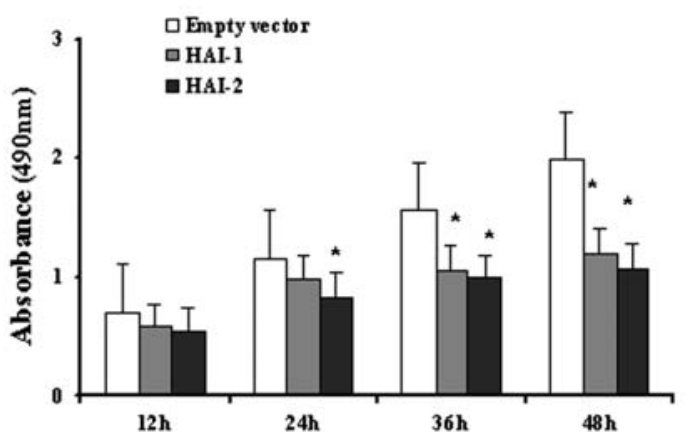

${ }^{*} \mathrm{p}<0.05$

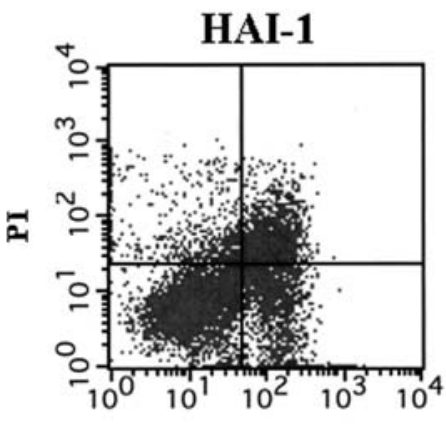

Annexin-V

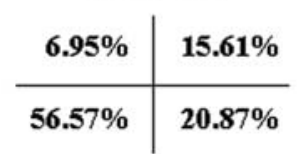

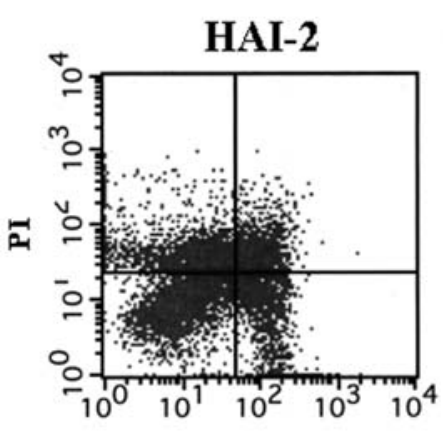

Annexin-V

\begin{tabular}{l|l}
$23.70 \%$ & $17.23 \%$ \\
\hline $37.12 \%$ & $21.95 \%$
\end{tabular}

Figure 4. (A) Matrigel invasion assay by HAI-1 or HAI-2 into OVCAR-3 ovarian cancer cells. Cells were plated at a density in 24-well plates, and were incubated for 12, 24, 36 and $48 \mathrm{~h}$. Following incubation, membranes were removed from the insert and mounted on slides, and the invading cells were counted under the microscope. Matrigel assays were performed in triplicate. (B) Cell Proliferation of MTS assay was analyzed in transient transfection with HAI-1 or HAI-2 for 12, 24, 36 and $48 \mathrm{~h}$ into OVCAR-3 ovarian cancer cells. The assays were carried out through quadruplate transfection experiments. (C) Representative flow cytometric data for apoptosis by transient transfection with HAI-1 or HAI-2 for 48 h in OVCAR-3 ovarian cancer cells.

transfection of HAI-1 and HAI-2 for $48 \mathrm{~h}$ showed increased FITC positive and Propidium iodide (PI) positive signal. HAI-1 and HAI-2 induced apoptosis in these cancer cells, which was assessed by Annexin V-FITC assay as shown in Fig. 4C. HAI-1 and HAI-2 respectively increased the total apoptotic and necrotic cells to $43.43 \%$ and $62.88 \%$ as compared with control of $6.92 \%$ in OVCAR-3 cells.

The protein expression of Bcl-2 and Bak by transient transfection with HAI-1 or HAI-2 into OVCAR-3 cell lines. We also investigated the effect of HAI-1 and HAI-2 on apoptotic molecules such as anti-apoptotic Bcl-2 and pro-apoptotic Bak on the protein level. As shown in Fig. 5A, the expression of Bak protein was up-regulated, while Bcl-2 was downregulated in the transient transfection with HAI-1 and HAI-2 in OVCAR-3 cell lines.

Inhibition of cell growth in a monolayer and in soft agar by transient transfection of HAI-1 or HAI-2 into OVCAR-3 cell lines. Effects of HAI-1 or HAI-2 expression on cell proliferation were analyzed using transient transfection of HAI-1 or HAI-2 into OVCAR-3 cell lines. We found significant inhibitory effect of HAI-1 or HAI-2 on cell growth in transient transfection of HAI-1 or HAI-2 into OVCAR-3 cell lines $(\mathrm{p}<0.05)$ as compared to the control (Fig. 5B). Furthermore, the transient transfected with HAI-1 or HAI-2 into OVCAR-3 cell lines resulted in a significantly reduced number of colonies in the colony formation assay when compared to the control $(\mathrm{p}<0.01)$ (Fig. 5C). Furthermore, we found a significant inhibitory effect of HAI-2 compared with HAI-1 on ovarian cancer.

\section{Discussion}

HAI-1 and HAI-2 are a Kunitz-type serine protease inhibitor that has a broad inhibitory spectrum against serine proteases, such as plasmin, trypsin, tissue, and plasma kallikreins and factor Xia. cDNA cloning of HAI-2 revealed that this protein was identical to placental bikunin (PB) (21). To date, several studies on HAI-1 and HAI-2 expression in ovarian cancer tissues have been published. The matriptase to HAI-1 ratio could be important in the development of advance stage in ovarian cancer (18). Tanaka and colleagues have reported that lowest HAI-2/PB expression may be associated with 
A 1. $2 . \quad 3$.

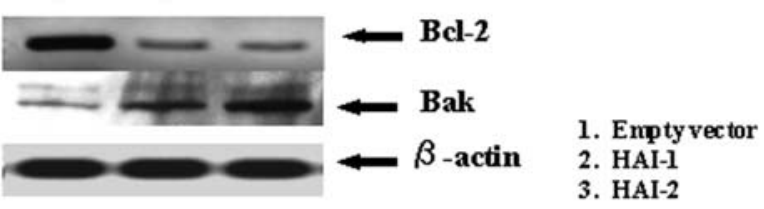

B

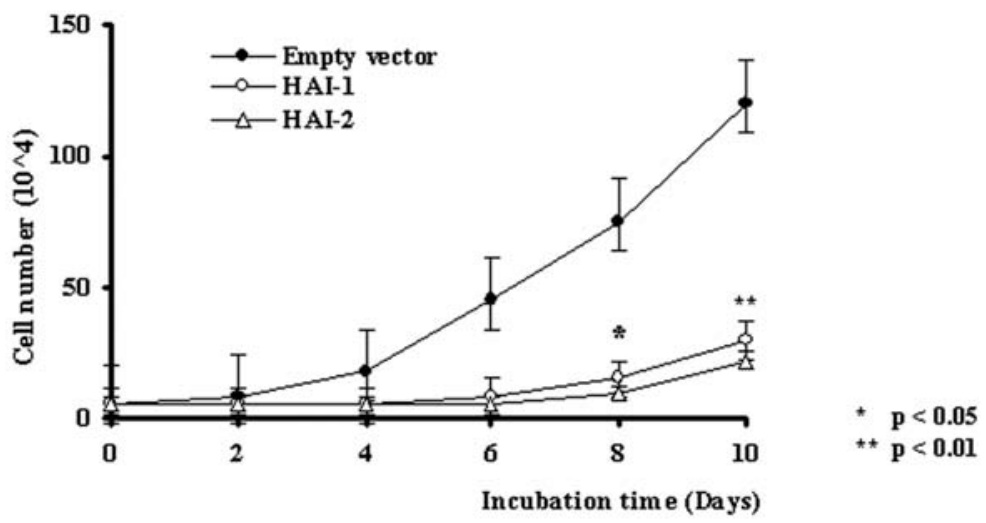

C

Colony formation on soft agar of transfectants transfection with HAI-1 or HAI-2 into OVCAR-3 ovarian cancer cells

\begin{tabular}{lrl}
\hline & Colonies/dish & $\begin{array}{c}\text { Inhibitory effect (\%) } \\
\text { (mean/SD)* }\end{array}$ \\
\hline Empty vector & $15.2 \pm 5.8$ & \multicolumn{1}{c}{0} \\
HAI-1 & $3.4 \pm 0.9$ & $77.6^{* *}$ \\
HAI-2 & $1.0 \pm 0.2$ & $94.5^{* * *}$ \\
\hline
\end{tabular}

* Numbers represent data fr om trip licate experim ents

$$
\begin{aligned}
& { }^{\star \star} \mathbf{p}<0.05 \\
& { }^{\star \star \star} \mathbf{p}<0.01
\end{aligned}
$$

Figure 5. Effects on the apoptotic pathway by HAI-1 or HAI-2 for $48 \mathrm{~h}$ in OVCAR-3 ovarian cancer cells. (A) Analyzed for Bak, Bcl-2 by Western blotting. B-actin antibody was used as loading control in the same blot. Effects on cell growth by HAI-1 or HAI- 2 for 48 h in OVCAR-3 ovarian cancer cells. (B) Monolayer growth of HAI-1 or HAI-2 in OVCAR-3 ovarian cancer cells incubated for 2, 4, 6, 8 and 10 days in DMEM with $10 \%$ FBS. Numbers represent data from triplicate experiments. (C) Colony formation on soft agar of HAI-1 or HAI-2 in OVCAR-3 ovarian cancer cells. Numbers represent data from triplicate experiments.

advanced stages, positive nodal status, and positive peritoneal status, and low HAI-2/PB expression correlates with poor prognosis in ovarian cancer (22). In the current study, we examined whether HAI- 1 and HAI-2 protein expression correlated with clinicopathological characteristics in patients suffering from ovarian cancer by immunohistochemistry. Immunohistochemistry on ovarian cancer tissues showed that HAI-1 expression associated with stage $(p=0.040)$. There was a significant correlation between HAI-2 expression and stage $(\mathrm{p}=0.031)$, amount of ascites $(p=0.002)$ and diameter of residual tumor size $(p=0.034)$ (Table I). Moreover, the low HAI-1 and HAI-2 expression was a significant predictor for poor prognosis compared with high HAI-1 and HAI-2 expression (DFS and OS rates: $\mathrm{p}=0.031, \mathrm{p}=0.003$ and $\mathrm{p}=0.048, \mathrm{p}=0.001$ ) (Fig. 2).

HAI-1 and HAI-2 are endogenous inhibitors of matriptase and hepsin. Both matriptase and hepsin are type II transmembrane proteins with an extracellular serine proteinase domain, and show enhanced expression in a variety of tumor tissues (23). Matriptase has been proposed to initiate signaling and proteolytic cascades through its ability to activate prourokinase (uPA) and protease-activated receptor 2 (PAR2). The $G$ protein-coupled receptor PAR2 and the plaminogen activator UPA are both activated by matriptase-mediated proteolysis in vitro, and some in vivo evidence exists to support the physiologic role of matriptase in activating these proteins $(24,25)$, and hepsin is thought to be involved in the activation of the coagulation cascade such as blood clotting factors VII, XII, and IX, and activate pro-uPA, and pro-HGF (26). Generation of plasmin from plasminogen by uPA can induce extracellular matrix degradation and promote tumor cell migration and metastasis. In this study, we investigated the mechanism that correlated between HAI-1, HAI-2, matriptase and hepsin in ovarian cancer cell lines. Both martipase and hepsin were significantly inhibited by HAI-1 and HAI-2 (Fig. 3A). However, the biological functions of HAI-1 and HAI-2 remain unknown. Previous reports also showed that HAI-2/PB acts during apoptosis $(27,28)$. In this study, we used MTS and FITC-conjugated Annexin V assays that examined apoptosis expression by transient transfection of 
HAI-1 or HAI-2 into OVCAR-3 ovarian cell lines. HAI-1 and HAI-2 induced apoptosis on OVCAR-3 ovarian cancer cells, as shown in Fig. 4B and C. To trace the steps in the apoptotic cascade, we evaluated the activation of the proapoptotic and anti-apoptotic proteins $(29,30)$. Our results indicated that HAI-1 and HAI-2 up-regulated the Bak proteins and down-regulated the Bcl-2 thereby inducing apoptosis (Fig. 5A). Overall, HAI-1 and HAI-2 show potential inhibitory effects mediated by reduction of matriptase and hepsin expression which leads to apoptosis through increasing the level of Bak and reducing the level of Bcl-2 in ovarian cancer. Therefore, we found a significant inhibitory effect of HAI-2 compared with HAI-1 on ovarian cancer in vitro and in vivo.

In summary, in vitro and in vivo studies revealed a critical role for HAI-1 and HAI-2 in disruption of the basement membrane of ovarian cancer. These findings identify HAI-1 and HAI-2 as a favorable prognostic marker, and could be considered a therapeutic target for treatment approaches in ovarian cancer.

\section{Acknowledgments}

This work was supported by cancer research grant of Hamazaki (K. Nakamura) and Ryobi Teien Memory Foundation 2007 (K. Nakamura). We are grateful to Dr H. Kataoka for providing the full-length HAI-1 and HAI-2 cDNA to the Section of Oncopathology and Regenerative Biology, Department of Pathology, Faculty of Medicine, University of Miyazaki, Japan.

\section{References}

1. Jeffers M, Rong S and Vande Woude GF: Enhanced tumorigenicity and invasion-metastasis by hepatocyte growth factor/ scatter factor-met signalling in human cells concomitant with induction of the urokinase proteolysis network. Mol Cell Biol 16: 1115-1125, 1996.

2. Comoglio PM: Structure, biosynthesis and biochemical properties of the HGF receptor in normal and malignant cells. EXS 65: 131-165, 1993.

3. Rosen EM, Nigam SK and Goldberg ID: Scatter factor and the c-Met receptor: a paradigm for mesenchymal/epithelial interactions. J Cell Biol 127: 1783-1787, 1994.

4. Ayhan A, Ertunc D, Tok EC and Ayhan A: Expression of the c-Met in advanced epithelial ovarian cancer and its prognostic significance. Int J Gynecol Cancer 15: 618-623, 2005 .

5. Sawada K, Radjabi AR, Shinomiya N, Kistner E, Kenny H, Becker AR, Turkyilmaz MA, Salgia R, Yamada SD, Vande Woude GF, Tretiakova MS and Lengyel E: c-Met overexpression is a prognostic factor in ovarian cancer and an effective target for inhibition of peritoneal dissemination and invasion. Cancer Res 67: 1670-1679, 2007

6. Shimomura T, Denda K, Kitamura A, Kawaguchi T, Kito M, Kondo J, Kagaya S, Qin L, Takata H, Miyazawa K and Kitamura N: Hepatocyte growth factor activator nhibitor, a novel Kunitz-type serine protease inhibitor. J Biol Chem 272: 6370-6376, 1997.

7. Kawaguchi T, Qin L, Shimomura T, Kondo J, Matsumoto K, Denda K and Kitamura N: Purification and cloning of hepatocyte growth factor activator inhibitor type 2, a Kunitz-type serine protease inhibitor. J Biol Chem 272: 27558-27564, 1997.

8. Kataoka H, Itoh H, Nuki Y, Hamasuna R, Naganuma S, Kitamura N and Shimomura T: Mouse hepatocyte growth factor (HGF) activator inhibitor type 2 lacking the first Kunitz domain potently inhibits the HGF activator. Biochem Biophys Res Commun 290: 1096-1100, 2002.
9. Müller-Pillasch F, Wallrapp C, Bartels K, Varga G, Friess H, Büchler M, Adler G and Gress TM: Cloning of a new Kunitztype protease inhibitor with a putative transmembrance domain overexpressed in pancreatic cancer. Biochim Biophys Acta 1395: 88-95, 1998.

10. Hamasuna R, Kataoka H, Meng JY, Itoh H, Moriyama T, Wakisaka S and Koono M: Reduced expression of hepatocyte growth factor activator inhibitor type-2/placental bikunin (HAI-2/PB) in human glioblastomas: implication for antiinvasive role of HAI-2/PB in glioblastoma cells. Int J Cancer 93: 339-345, 2001.

11. Kataoka H, Shimomura T, Kawaguchi T, Hamasuna R, Itoh H, Kitamura N, Miyazawa K and Koono M: Hepatocyte growth factor activator inhibitor type 1 is a specific cell surface binding protein of hepatocyte growth factor activator (HGFA) and regulates HGFA activity in the pericellular microenvironment. J Biol Chem 275: 40453-40462, 2000.

12. Lin CY, Anders J, Johnson M and Dickson RB: Purification and characterization of a complex containing matriptase and a Kunitz-type serine protease inhibitor from human milk. J Biol Chem 274: 18237-18242, 1999.

13. Kataoka H, Hamasuna R, Itoh H, Kitamura $\mathrm{N}$ and Koono M: Activation by hepatocyte growth factor/scatter in colorectal carcinoma. Cancer Res 60: 6148-6159, 2000.

14. Parr C, Watkins G, Mansel RE and Jiang WG: The hepatocyte growth factor regulatory factors in human breast cancer. Clin Cancer Res 10: 202-211, 2004.

15. Saleem M, Adhami VM, Zhong W, Longley BJ, Lin CY, Dickson RB, Reagan-Shaw S, Jarrard DF and Mukhtar H: A novel biomarker for staging human prostate adenocarcinoma: overexpression of matriptase with concomitant loss of its inhibitor, hepatocyte growth factor activator inhibitor-1. Cancer Epidemiol Biomarkers Prev 15: 217-227, 2006.

16. Nagaike K, Kohama K, Uchuyama S, Tanaka H, Chijiiwa K, Itoh $\mathrm{H}$ and Kataoka $\mathrm{H}$ : Paradoxically enhanced immunoreactivity of hepatocyte growth factor activator inhibitor type 1 (HAI-1) in cancer cells at the invasion front. Cancer Sci 95: 728-735, 2004.

17. Yamauchi M, Kataoka H, Itoh H, Seguchi T, Hasui Y and Osada Y: Hepatocyte growth factor activator inhibitor types 1 and 2 are expressed by tubular epithelium in kidney and down-regulated in renal cell carcinoma. J Urol 171: 890-896, 2004.

18. Oberst MD, Johnson MD, Dickson RB, Lin CY, Singh B, Stewart M, Williams A, Al-Nafussi A, Smyth JF, Gabra H and Sellar GC: Expression of the serine protease matriptase and its inhibitor HAI-1 in epithelial ovarian cancer: correlation with clinical outcome and tumor clinicopathological parameters. Clin Cancer Res 8: 1101-1107, 2002.

19. Fukai K, Yokosuka O, Chiba T, Hirasawa Y, Tada M, Imazeki F, Kataoka $\mathrm{H}$ and Saisho $\mathrm{H}$ : Hepatocyte growth factor activator inhibitor 2/placental bikunin HAI-2/PB gene is frequently hypermethylated in human hepatocellular carcinoma. Cancer Res 63: 8674-8679, 2003.

20. Betsunoh H, Mukai S, Akiyama Y, Fukushima T, Minamiguchi N, Hasui Y, Osada Y and Kataoka H: Clinical relevance of hepsin and hepatocyte growth factor activator inhibitor type 2 expression in renal cell carcinoma. Cancer Sci 98: 491-498, 2007.

21. Marlor CW, Delaria KA, Davis G, Muller DK, Greve JM and Tamburini PP: Identification and cloning of human placental bikunin, a novel serine protease inhibitor containing two kunitz domains. J Biol Chem 272: 12202-12208, 1997.

22. Tanaka Y, Kobayashi H, Suzuki M, Kanayama N, Suzuki M and Terao T: Upregulation of bikunin in tumor-infiltrating macrophages as a factor of favorable prognosis in ovarian cancer. Gynecol Oncol 94: 725-734, 2004.

23. Netzel-Arnett S, Hooper JD, Szabo R, Madison EL, Quigley JP, Bugge TH and Antalis TM: Membrane anchored serine proteases: a rapidly expanding group of cell surface proteolytic enzymes with potential roles in cancer. Cancer Metast Rev 22: 223-236, 2003 .

24. Lee SL, Dickson RB and Lin CY: Activation of hepatocyte growth factor and urokinase/plasminogen activator by matriptase, an epithelial membrane serine protease. J Biol Chem 275: 36720-36725, 2000.

25. Takeuchi T, Harris JL, Huang W, Yan KW, Coughlin SR and Craik CS: Cellular localization of membrane-type serine protease 1 and identification of protease-activated receptor-2 and single-chain urokinase-type plasminogen activator as substrates. J Biol Chem 275: 26333-26342, 2000. 
26. Kazama Y, Hamamoto T, Foster DC and Kisiel W: Hepsin, a putative membrane-associated serine protease, activates human factor VII and initiates a pathway of blood coagulation on the cell surface leading to thrombin formation. J Biol Chem 270: 66-72, 1995.

27. Suzuki M, Kobayashi H, Tanaka Y, Hirashima Y, Kanayama N, Takei Y, Saga Y, Suzuki M, Itoh H and Terao T: Bikunin target genes in ovarian cancer cells identified by microarray analysis. J Biol Chem 278: 14640-14646, 2003.

28. Suzuki M, Kobayashi H, Tanaka Y, Kanayama N and Terao T: Reproductive failure in mice lacking inter-alpha-trypsin inhibitor (ITI)-ITI target genes in mouse ovary identified by microarray analysis. J Endocrinol 183: 29-38, 2004.
29. Krajewska M, Moss SF, Krajewski S, Song K, Holt PR and Reed JC: Elevated expression of Bcl-X and reduced Bak in primary colorectal adenocarcinomas. Cancer Res 56: 2422-2427, 1996.

30. Yamamoto K, Ichijo $\mathrm{H}$ and Korsmeyer SJ: BCL-2 is phosphorylated and inactivated by an ASK1/Jun N-terminal protein kinase pathway normally activated at $\mathrm{G}(2) / \mathrm{M}$. Mol Cell Biol 19: 8469-8478, 1999. 